\title{
A CHARACTERIZATION FOR THE PRODUCT OF CLOSED IMAGES OF METRIC SPACES TO BE A $k$-SPACE
}

\author{
YOSHIO TANAKA
}

\begin{abstract}
We give, under [ $\mathrm{CH}]$, a necessary and sufficient condition for the product of two closed images of metric spaces to be a $k$-space.
\end{abstract}

1. Introduction. In [14, Theorem 4.3], we proved the following result. Recall that a space $X$ is said to belong to class $\mathfrak{I}^{\prime}$ if it is the union of countably many closed and locally compact subsets $X_{n}$ such that $F \subset X$ is closed whenever $F \cap X_{n}$ is closed for all $n$.

TheOREM 1.0. Let $X$ and $Y$ be closed s-images of metric spaces. Then $X \times Y$ is a $k$-space if and only if one of the following three properties holds:

(1) $X$ and $Y$ are metrizable spaces.

(2) $X$ or $Y$ is a locally compact, metrizable space.

(3) $X$ and $Y$ are spaces of class $\mathfrak{I}^{\prime}$.

In that place, we raised the question whether this theorem remains true if "s-images" is weakened to "images".

In this paper, under the continuum hypothesis $[\mathrm{CH}]$, we shall give the following affirmative answer to this question.

THEOREM $1.1[\mathrm{CH}]$. Let $X$ and $Y$ be closed images of metric spaces under maps $f$ and $g$ respectively. Then $X \times Y$ is a $k$-space (equivalently, $f \times g$ is a quotient map by [6, Theorem 1.5]) if and only if one of the three properties of Theorem 1.0 holds.

Throughout this paper, we shall assume that all spaces are regular $T_{2}$, and all maps are continuous surjections.

2. Preliminaries. A space $X$ is Fréchet if, whenever $x \in \bar{A}$, then some sequence of points of $A$ converges to $x$. Obviously, every closed image of a first countable space is Fréchet.

Recall that a space $X$ is strongly Fréchet [10] (= countably bi-sequential in the sense of E. Michael [7]) if, whenever $\left\{F_{n} ; n=1,2, \ldots\right\}$ is a decreasing sequence accumulating at $x \in X$, there exist $x_{n} \in F_{n}$ such that the sequence $\left\{x_{n} ; n=1,2, \ldots\right\}$ converges to $x$. Clearly every strongly Fréchet space is Fréchet.

Received by the editors November 15, 1977 and, in revised form, June 8, 1978.

AMS (MOS) subject classifications (1970). Primary 54B10, 54D50; Secondary 54C10, 54E35. $K e y$ words and phrases. $k$-spaces, Fréchet spaces, strongly Fréchet spaces, Lindelöf spaces. 
LEMMA 2.1 (cf. [7, THEOREM 9.9]). Let $X$ be the closed image of a metric space (more generally, paracompact space) under a map $f$. If $X$ is strongly Fréchet, then $\partial f^{-1}(x)$ is compact for every $x \in X$.

Since every Fréchet space is a sequential space, by [13, Lemma 2.1 (A) and Proposition 2.4] and [12, Theorem 2.2], we have

Lemma 2.2. Let $X$ be a Fréchet space, and let $Y$ be a metric space. Suppose that $X \times Y$ is a $k$-space. Then $X$ is strongly Fréchet, or $Y$ is locally compact.

LEMMA 2.3. Let $X$ be a Fréchet space, or a $k$-space each of whose points is a $G_{\delta}$-set. Let $Y$ be the closed image of a collectionwise normal and Fréchet space $Z$ under a map $f$. Suppose that $X \times Y$ is a $k$-space. Then $X$ is strongly Fréchet, or every $\partial f^{-1}(y)$ has property $(\mathrm{P})$ below.

(P) Every subset of cardinality $2^{\kappa_{0}}$ in $\partial f^{-1}(y)$ has an accumulation point.

Proof. Suppose that there is $y_{0} \in Y$ such that $\partial f^{-1}\left(y_{0}\right)$ does not have property (P). Then there is a closed discrete subset $\left\{x_{\alpha} ; \alpha \in A\right\}$ of $\partial f^{-1}\left(y_{0}\right)$ with $|A|=2^{\kappa_{0}}$. Since $Z$ is collectionwise normal, there is a discrete open collection $\left\{U_{\alpha} ; \alpha \in A\right\}$ in $Z$ with $x_{\alpha} \in U_{\alpha}$. Since $Z$ is Fréchet, and $x_{\alpha} \in \overline{U_{\alpha}-f^{-1}\left(y_{0}\right)}$ for each $\alpha \in A$, then there is a convergent sequence $\left\{x_{\alpha i} ; i=1,2, \ldots\right\}$ of $U_{\alpha}-f^{-1}\left(y_{0}\right)$ with its limit point $x_{\alpha}$. Let $C_{\alpha}=\left\{x_{\alpha i}\right.$; $i=1,2, \ldots\} \cup\left\{x_{\alpha}\right\}$ for each $\alpha \in A$, and let $Z_{0}=\cup_{\alpha \in A} C_{\alpha}$. Then, since $\left\{C_{\alpha} ; \alpha \in A\right\}$ is a discrete closed collection in $Z, Z_{0}$ is a closed subset of $Z$. Let $g=f \mid Z_{0}$. Then $g$ is a closed map from the locally compact, metric space $Z_{0}$. Let $Y_{1}=\left\{y \in Y_{0} ; g^{-1}(y)\right.$ is not compact $\}$, where $Y_{0}=g\left(Z_{0}\right)$. Then, by [8, Theorem 4], $Y_{1}$ is a closed discrete subset of $Y_{0}$. It is easy to see that $y_{0} \in Y_{1}$. Since the sequence $g\left(C_{\alpha}\right)$ converges to $y_{0}$, and $Y_{1}$ is closed and discrete, then each $C_{\alpha}$ intersects only a finite number of $g^{-1}(y), y \in Y_{1}$. Hence $C_{\alpha}^{\prime}=C_{\alpha}-g^{-1}\left(Y_{1}\right)$ is infinite, which implies that each sequence $C_{\alpha}^{\prime}$ converges to $x_{\alpha}$. For each $\alpha \in A$, let $A_{\alpha}=g\left(C_{\alpha}^{\prime}\right)$. Then $\mathfrak{A}=\left\{A_{\alpha} ; \alpha \in A\right\}$ is locally finite, hence point-finite in $Y_{0}-Y_{1}$. For, $g$ is a perfect map on $Z_{0}^{\prime}=Z_{0}-g^{-1}\left(Y_{1}\right)$ and $\left\{C_{\alpha}^{\prime} ; \alpha \in A\right\}$ is a discrete collection in $Z_{0}^{\prime}$. Since each $A_{\alpha}$ is countable, for each $\alpha \in A, A(\alpha)=\left\{\beta \in A ; A_{\alpha} \cap A_{\beta} \neq \varnothing\right\}$ is at most countable. Then, there is a subset $A^{\prime}$ of $A$ with cardinality $2^{\mathrm{N}_{0}}$, such that $\mathfrak{U}^{\prime}=\left\{A_{\alpha} ; \alpha \in A^{\prime}\right\}$ is pairwise disjoint. Indeed, let $A=\left\{\alpha ; \alpha<2^{\omega_{0}}\right\}$. Then, for each $\alpha$, there is a pairwise disjoint subcollection $\mathfrak{B}_{\alpha}$ of $\mathfrak{A}$ such that $\left|\mathfrak{B}_{\alpha}\right| \leqslant|\alpha|$ and $\cup_{\beta<\alpha} \mathfrak{B}_{\beta} \subsetneq \mathfrak{B}_{\alpha}$. For, let $\left\{\mathfrak{B}_{\beta} ; \beta<\alpha\right\}$ be defined for each $\beta<\alpha$. Then we can choose $A_{\alpha^{\prime}} \in \mathfrak{U}$ with

$$
A_{\alpha^{\prime}} \cap\left(\bigcup_{\beta<\alpha}\left\{A_{\delta} ; A_{\delta} \in \mathfrak{B}_{\beta}\right\}\right)=\varnothing,
$$

for each $A(\delta)$ is at most countable and $\left|\cup_{\beta<\alpha} \mathfrak{B}_{\beta}\right|<|\alpha|\left(\neq 2^{\boldsymbol{\kappa}_{0}}\right)$. Let $\mathfrak{B}_{\alpha}=\left\{A_{\alpha^{\prime}}\right\} \cup \cup_{\beta<\alpha} \mathfrak{B}_{\beta}$. Then $\mathfrak{B}_{\alpha}$ satisfies the conditions. Hence, $\mathfrak{U}^{\prime}=$ $\cup_{\alpha<2^{\omega}{ }^{\omega}} \mathfrak{B}_{\alpha}$ is a pairwise disjoint subcollection of $\mathfrak{A}$ with cardinality $2^{\aleph_{0}}$. 
Now, let $Z_{1}=\cup_{\alpha \in A^{\prime}}\left\{C_{\alpha}^{\prime} \cup\left\{x_{\alpha}\right\}\right\}$. Let $h=f \mid Z_{1}$. Then, since $Z_{1}$ is closed in $Z, h$ is a closed map, hence is quotient. Moreover, $h\left(x_{\alpha}\right)=y_{0}$ for each $\alpha \in A^{\prime}$ and $h$ is one-to-one on $\cup_{\alpha \in A^{\prime}} C_{\alpha}^{\prime}$ by the choice of the index set $A^{\prime}$. Here, we may assume that $h \mid C_{\alpha}^{\prime}$ is one-to-one for each $\alpha \in A^{\prime}$. Thus, $h\left(Z_{1}\right)$ can be shown to be homeomorphic to a quotient space $Z_{1} / F_{1}$ obtained from $Z_{1}$ identifying all points of $F_{1}=h^{-1}\left(y_{0}\right)$.

On the other hand, $X \times h\left(Z_{1}\right)$ is a closed subset of a $k$-space $X \times Y$, for $h\left(Z_{1}\right)$ is closed in $Y$. Hence $X \times h\left(Z_{1}\right)$ is a $k$-space. This implies that $X \times\left(Z_{1} / F_{1}\right)$, which is homeomorphic to $X \times h\left(Z_{1}\right)$, is a $k$-space. Thus, by [15, Lemma 2.1(2)], $X$ is strongly Fréchet or $\partial_{Z_{1}} F_{1}$ has property (P). However, $\partial_{Z_{1}} F_{1}$ contains a closed discrete subset $\left\{x_{\alpha} ; \alpha \in A^{\prime}\right\}$ of cardinality $2^{\alpha_{0}}$. Then it does not have property (P). Therefore $X$ is strongly Fréchet. That completes the proof.

Proposition 2.4 [CH]. Let $X$ be a Fréchet space, or a $k$-space each of whose points is $a G_{\delta}$-set. Let $Y$ be the closed image of a first countable, paracompact space under a map f. If $X \times Y$ is a $k$-space, then either $X$ is strongly Fréchet, or $\partial f^{-1}(y)$ is locally compact and Lindelöf for every $y \in Y$.

Proof. Suppose that $X$ is not strongly Fréchet. Then, without [CH], every $\partial f^{-1}(y)$ is locally compact by [15, Theorem 2.2]. Moreover, from Lemma 2.3, every $\partial f^{-1}(y)$ has property $(\mathrm{P})$. Then, under $[\mathrm{CH}]$ it is easy to see that every $\partial f^{-1}(y)$ is Lindelöf, for every $\partial f^{-1}(y)$ is paracompact.

\section{Proof of Theorem 1.1 and a related result.}

Proof of Theorem 1.1. The "if" part is that of Theorem 1.0 stated in $\$ 1$. So we shall prove the "only if" part.

(i) Suppose that every $\partial f^{-1}(x)$ is Lindelöf: If every $\partial g^{-1}(y)$ is also Lindelöf, as in the proof of [5, Corollary 1.2], we may assume that $X$ and $Y$ are closed $s$-images of metric spaces. Thus, by the "only if" part of Theorem 1.0, the assertion holds. If some $\partial g^{-1}\left(y_{0}\right)$ is not Lindelöf, then $X$ is strongly Fréchet by Proposition 2.4. Thus $X$ is metrizable by Lemma 2.1. On the other hand, $Y$ is not strongly Fréchet by Lemma 2.1, for $\partial g^{-1}\left(y_{0}\right)$ is not compact. Hence $X$ is locally compact by Lemma 2.2 .

(ii) Suppose that some $\partial f^{-1}\left(x_{0}\right)$ is not Lindelöf: Then, as above, $Y$ is locally compact and metrizable. That completes the proof.

As for the product of closed images of locally compact metric spaces, we have the following theorem, which is an improvement of [15,Proposition 2.6 or 2.7]. The "only if" part follows from the proof of Theorem 1.1. The "if" part follows from Proposition 3.2 below.

THEOREM $3.1[\mathrm{CH}]$. Let $f_{i}: X_{i} \rightarrow Y_{i}(i=1,2)$ be closed maps such that each $X_{i}$ is a locally compact metric space (more generally, locally compact, Fréchet and paracompact space). Then $Y_{1} \times Y_{2}$ is a $k$-space if and only if either of the following properties holds: 
(1) Every $\partial f_{1}^{-1}\left(y_{1}\right)$ is compact, or every $\partial f_{2}^{-1}\left(y_{2}\right)$ is compact. (Hence, $Y_{1}$ or $Y_{2}$ is locally compact.)

(2) Every $\partial f_{i}^{-1}\left(y_{i}\right)$ is Lindelöf for $i=1,2$.

Proposition 3.2. (a) [4, Theorem 3.2] Let $Y_{1}$ be a $k$-space, and let $Y_{2}$ be a locally compact space. Then $Y_{1} \times Y_{2}$ is a $k$-space.

(b) [15, Lemma 2.5] Let $Y_{i}(i=1,2)$ be closed images of locally compact spaces under maps $f_{i}$ with each $\partial f_{i}^{-1}\left(y_{i}\right)$ Lindelöf. Then $Y_{1} \times Y_{2}$ is a $k$-space.

\section{Some remarks to Theorem 1.1.}

REMARK 4.1. Concerning the "Fréchetness" for the product of two closed images of metric spaces, we have the following theorem from [9, Theorem 9.2] (also cf. [7, Proposition 4.D.5]), together with Lemma 2.1.

TheOREM. Let $X$ and $Y$ be closed images of metric spaces. Then $X \times Y$ is a Fréchet space (equivalently, hereditary $k$-space by [2]) if and only if either of the following properties holds:

(1) $X$ and $Y$ are metrizable spaces.

(2) $X$ or $Y$ is a discrete space.

REMARK 4.2. Concerning the " $k$-ness" for the product of countably many copies of a closed image of a metric space, we have the following theorem from [13, Theorem 1.3] and [7, Theorem 7.3].

THEOREM. Let $X$ be a closed image of a metric space. Then $X^{\omega}$ is a $k$-space if and only if $X$ is a metrizable space.

REMARK 4.3. As generalizations of metric spaces, J. G. Ceder [3] introduced three types of topological spaces which he called $M_{1}, M_{2}$ and $M_{3}$-spaces, and observed that $M_{1} \Rightarrow M_{2} \Rightarrow M_{3}$. An $M_{1}$-space is a regular space having a $\sigma$-closure preserving base. That every closed image of a metric space is $M_{1}$ was proved by F. Slaughter [11]. The following example shows that Theorem 1.1 becomes false if "closed images of metric spaces" is weakened to " $M_{1}$ spaces", even if in property (1) of Theorem 1.0 we replace "metrizable spaces" by "first countable spaces".

EXAMPLE. Let $X$ be the Nagata space constructed in Example 9.2 in [3] $(X=\{(x, y) ; 0<x<1, y \geqslant 0\}$ : the topology on $X$ has a base consisting of disks missing the $x$-axis and sets of the form $U_{n}(p)=\{p\} \cup\{(x, y) ;|x-p|$ $<1 / n$ and $y$ lies below the graph of $\left.\left.(x-p)^{2}+(y-n)^{2}=n^{2}\right\}\right)$. Obviously $X$ is separable, first countable and not second countable. Hence $X$ is not metrizable. The proof that $X$ is $M_{1}$, which is due to J. Nagata, is given in [3]. Let $C$ be a closed interval contained in $(0,1)$. Let $Y$ be a quotient space obtained by identifying all points of $C$, and let $f: X \rightarrow Y$ be the natural quotient map. Since $C$ is compact in $X, f$ is a perfect map. Then $Y \times Y$ is a $k$-space, for it is the perfect image of a first countable space $X \times X$. To show that $Y$ is $M_{1}$, let $\mathscr{B}=\cup_{i=1}^{\infty} \mathfrak{B}_{i}$ be a $\sigma$-closure preserving base for $X$. We may assume that $\mathfrak{B}_{i} \subset \mathfrak{B}_{i+1}$ for each $i$, and that each $\mathfrak{B}_{i}$ is closed under arbitrary 
unions. Then, since $C$ is compact in $X,\{f(B) ; B \in \mathfrak{B}$ with $C \subset B$ or $C \cap B=\varnothing\}$ is a $\sigma$-closure preserving base for $Y$.

That $Y$ is not first countable will be shown below, hence neither is $Y$ locally compact by [3, Corollary 5.7]. Suppose that $Y$ is first countable. Then the compact, separable metric subset $C$ is of countable character in $X$ (Arhangel'skii [1, Definition 3.5]). Then, by [1, Lemma 3.2], there is a countable collection $\mathfrak{B}$ of open subsets of $X$ such that, if $c \in C$ and $c \in U$ with $U$ open in $X$, then $c \in V \subset U$ for some $V \in \mathfrak{B}$. This implies that a subspace $C \times\{y ; y \geqslant 0\}$ of $X$ is second countable. But this is a contradiction, for the subspace is obviously non-second countable.

To show that $Y$ is not a space of class $\mathfrak{I}^{\prime}$, suppose not. Then $Y$ is the union of countably many closed and locally compact subsets $Y_{n}$ such that, $F \subset Y$ is closed whenever $F \cap Y_{n}$ is closed for each $n$. We may assume that $Y_{n} \subset Y_{n+1}$ for each $n$. Then each compact subset of $Y$ is contained in some $Y_{n}$. For any $x \in X$, let $\left\{V_{n} ; n=1,2, \ldots\right\}$ be a decreasing local base at $x$. Then, for some $m, f\left(V_{m}\right) \subset Y_{m}$, hence $V_{m} \subset f^{-1}\left(Y_{m}\right)$. While, since $f$ is perfect, $f^{-1}\left(Y_{m}\right)$ is locally compact. Hence, by [3, Corollary 5.7], $f^{-1}\left(Y_{m}\right)$ is metrizable, so is $V_{m}$. This implies that $X$ is a locally metrizable space. Then $X$ is metrizable, for it is Lindelöf. But, this is a contradiction to the fact that $X$ is nonmetrizable. Thus $Y$ is not a space of class $\mathfrak{I}^{\prime}$.

\section{REFERENCES}

1. A. V. Arhangel'skii, Bicompact sets and the topology of spaces, Trans. Moscow Math. Soc. 13 (1965), 1-62.

2. A A characterization of very $k$-spaces, Czechoslovak Math. J. 18 (93) (1968), 392-395.

3. J. Ceder, Some generalizations of metric spaces, Pacific J. Math. 11 (1961), 105-125.

4. D. E. Cohen, Spaces with weak topology, Quart. J. Math. Oxford Ser. (2) 5 (1954), 77-80.

5. E. Michael, $A$ note on closed maps and compact sets, Israel J. Math. 2 (1964), 173-176.

6. , Bi-quotient maps and Cartesian products of quotient maps, Ann. Inst. Fourier (Grenoble) 18 (1968), fasc. 2, 287-302.

7. A quintuple quotient quest, General Topology and Appl. 2 (1972), 91-138.

8. K. Morita, On closed mappings, Proc. Japan Acad. 32 (1956), 539-543.

9. Some results on M-spaces, (Topics in topology, Colloq. Math. Societatis János Bolyai, Vol. 8, Keszthely, Hungary, 1972), North-Holland, Amsterdam, 1974, pp. 489-503.

10. F. Siwiec, Sequence-covering and countably bi-quotient mappings, General Topology and Appl. 1 (1971), 143-154.

11. F. Slaughter, The closed image of metrizable space is $M_{1}$, Proc. Amer. Math. Soc. 37 (1973), 309-314.

12. Y. Tanaka, On quasi-k-spaces, Proc. Japan Acad. 46 (1970), 1074-1079.

13. __ Products of sequential spaces, Proc. Amer. Math. Soc. 54 (1976), 371-375.

14. $\ldots$ characterization for the products of $k$-and- $\aleph_{0}$-spaces and related results, Proc. Amer. Math. Soc. 59 (1976), 149-155.

15. On the $k$-ness for the products of closed images of metric spaces, General Topology and Appl. 9 (1978), 175-183.

Department of Mathematics, Tokyo Gakugei University, 4-1-1 Nukuikita-machi, KoganeI-SHI, TOKYO, JAPAN 